\section{ESTIMATING THE PREVALENCE OF OCCUPATIONAL EXPOSURE IN LUNG CANCER. RESULTS FROM AN HOSPITAL-BASED CASE-SERIES IN ITALY}

\begin{abstract}
${ }^{1,2} \mathrm{M}$ Bonzini, 'L Bordini, ${ }^{2} \mathrm{~V}$ Paticchia, ${ }^{2} \mathrm{Z}$ Zucca, ${ }^{3} \mathrm{C}$ Bareggi, ${ }^{4} \mathrm{~A}$ Palleschi, ${ }^{1} \mathrm{D}$ Consonni, ${ }^{1,2} \mathrm{AC}$ Pesatori, 'L Riboldi. ' Occupational Medicine, Fondazione IRCCS Cà Granda Ospedale Maggiore Policlinico, Milan, Italy; ${ }^{2}$ Department of Clinical Sciences and Community Health, University of Milan, Italy; ${ }^{3}$ Medical Oncology, Fondazione IRCCS Ca' Granda Ospedale Maggiore Policlinico, Milan, Italy; ${ }^{4}$ Division of Thoracic Surgery, Fondazione IRCCS Ca' Granda Ospedale Maggiore Policlinico, Milan, Italy
\end{abstract}

\subsection{6/oemed-2018-ICOHabstracts.1245}

Introduction Lung cancer (LC) is the leading oncologic cause of death among males. The role of occupational history with exposure to well-established carcinogens is very important, but usually deeply underestimated. To undervalue the occupational origin of cancer can affect the efficacy of preventive measures and preclude patients to receive insurance benefits.

We performed a systematic occupational medicine evaluation of a cases-series of lung cancer cases to properly quantify the proportion of LC cases with previous occupational exposure to carcinogens.

Methods We systematically evaluated all consecutive LC cases hospitalised in a large university hospital, in Milan. An active systematic search was carried out for 24 months, by trained occupational physicians, using a standardised questionnaire.

Results We collected 123 consecutive LC cases (66\% males). Former and current smoking habit was found in $61 \%$ and $22 \%$, respectively. A clear exposure to occupational carcinogens (in details: asbestos, polycyclic aromatic hydrocarbons, paintings, diesel exhaust) was recognised in $10 \%$ of males cases.

Discussion Previous occupational exposure to carcinogens was frequent among males cases in Northern Italy. The observed prevalence was even lower than the one reported in a previous systematic search in Lombardy (Porru S, et al. Int Arch Occ Env Health 89:981-9). If we applied our observed proportion of occupational cases to the entire incident LC cases in Lombardy population, we should observe about 460 male occupational LC cases per year (AIRTUM-AIOM report 2016), actually more than the entire number of cancer (all sites, both gender) annually notified to National Institute for Insurance (INAIL Rapporto regionale 2015).

\section{SPIROERGOMETRY EXAMINATION IN PATIENT WITH LUNG SILICOSIS}

M Varga, T Takácsová. Department of Occupational Diseases and Clinical Toxicology University hospital L. Pasteur and Faculty of Medicine University of P. J. Safarik, Košice, Slovakia

\subsection{6/oemed-2018-ICOHabstracts.1246}

Introduction The purpose of the study was to compare lung function tests in patients with occupational interstitial lung disease (silicosis) and patients with airways diseases (COPD, asthma) by spiroergometry examination and spirometry, and to find diferences between groups.

Methods From 2005 to 2016, one hundred and fifty-five patients underwent spiroergometry and lung function testing. The sample of patients was male (143) and female (12) who were or have been exposed to hazardous work with exposure to solid fibrogenic aerosol. From the sample we exclude 6 patients with hypersensitive pneumonitis. Patients sample we split into 3 groups: the first is represented by patients with silicosis (50), where we included patients with simple and complicated silicosis; the second group represents patients with airways disease (chronic obstructive pulmonary disease, asthma and chronic bronchitis - 52) and the last group consists of a comparative sample of patients without pulmonary or respiratory disease (47). Spiroergometry was performed by a bicycle spiroergometer with gas analysis (O2 and $\mathrm{CO} 2)$. The test was done by ramp-type load. The termination of the examination depended on the patient's abilities.

Results Oxygen consuption (VO2 - Lt/min.) at rest, at anaerobic threshold (AT) and at maximal load (MAX.) show no statistical significant diferences (Rest: 1.gr. 0,37 vs 2.gr. 0,35 vs 3.gr. 0,36; AT: 1. gr. 1,12 vs 2 .gr. 1,25 vs 3.gr. 1,24; MAX: 1 .gr. 1,66 vs 2 .gr. $1,88$ vs 3.gr. 2,0$)$. We found only statistical diference $(p=0,044)$ between groups in oxygen consumption per kilogram of weight (VO2 $/ \mathrm{kg}-\mathrm{ml} / \mathrm{kg} / \mathrm{min}$.) at maximal load (1.gr. 19,23 vs 2.gr. 22,07 vs 3.gr. 23,35). Exhaled carbon dioxide (VCO2- Lt/min.) show statistical significant values $(\mathrm{p}=0,018)$ between groups at maximum load (1.gr. 1,82 vs 2 .gr. 2,1 vs 3 .gr. 2,33 ). There were statistical significant differences at respiratory parameters (BR-breath reserve, VD/VT ratio).

Discussion Parameters VO2/kg-max and VCO2-max can be very valuable and useful for diagnosis and prognosis of the patient. Benini (2017) suggests that patients with significantly reduced oxygen consumption per kilogram of weight have an increased risk of death and overall impaired exercise tolerance. Also abnormal ventilation response during an exercise test is associated with worse survival.

\section{A RETROSPECTIVE COHORT STUDY OF CANCER MORTALITY IN EMPLOYEES OF A RUSSIAN CHRYSOTILE ASBESTOS MINE AND MILLS: UPDATE 2017}

${ }^{1} \mathrm{EV}$ Kovalevskiy*, ${ }^{2,3} \mathrm{SJ}$ Schonfeld, ${ }^{2,4} \mathrm{E}$ Feletto, ${ }^{5} \mathrm{SV}$ Kashanskiy, ${ }^{2} \mathrm{M}$ Moissonier, ${ }^{2} \mathrm{E}$ Ostroumova, ${ }^{2} \mathrm{D}$ Hashim, ${ }^{6} \mathrm{~K}$ Straif, ${ }^{7} \mathrm{H}$ Kromhout, ${ }^{1} \mathrm{IV}$ Bukhtiyarov, ${ }^{2} \mathrm{~J}$ Schüz. ${ }^{1} / \mathrm{zmerov}$ Research Institute of Occupational Health, Moscow, Russian Federation; '2Section of Environment and Radiation, International Agency for Research on Cancer, Lyon, France; ${ }^{3}$ Division of Cancer Epidemiology and Genetics, National Cancer Institute, National Institutes of Health, Bethesda, MD, USA; ${ }^{4}$ Cancer Research Division, Cancer Council New South Wales, Woolloomooloo, Australia; ${ }^{5}$ Yekaterinburg Medical Research Centre for Prophylaxis and Health Protection in Industrial Workers, Yekaterinburg, Russian Federation; ${ }^{6}$ Section of IARC Monographs, International Agency for Research on Cancer, Lyon, France; ${ }^{7}$ Institute for Risk Assessment Sciences, Utrecht University, Utrecht, The Netherlands

10.1136/oemed-2018-ICOHabstracts. 1247

Introduction A retrospective cohort study of employees of one of the world's largest chrysotile asbestos mine and mills (JSC Uralasbest, Asbest, Russia) is presently being conducted. The primary aim of the study is to more precisely characterise and quantify the exposure-response relationship for total and sitespecific cancer risks associated with chrysotile exposure.

Methods The study includes workers employed for at least one year during 1975-2010 in the mine, enrichment factories, transportation, laboratory, and the explosives unit. Ascertainment of cohort members through extraction of occupational histories from the enterprises' archives started in 2012.

Results At present, the cohort comprises more than 35000 workers. A detailed occupational history was collected for each cohort member. Temporal trends in exposure levels were analysed using a database containing 89290 monthly-averaged gravimetric dust concentrations from stationary sampling points in the enrichment factories (1951-2001) and 1457 in the mine (1964-2001). Parallel gravimetric and fibre (PCOM) 